ORIGINAL ARTICLE

\title{
Effect of voluntary alcohol consumption on Maoa expression in the mesocorticolimbic brain of adult male rats previously exposed to prolonged maternal separation
}

\author{
M Bendre ${ }^{1}$, E Comasco $^{1}$, I Nylander ${ }^{2}$ and KW Nilsson ${ }^{3}$
}

Discordant associations between monoamine oxidase $\mathrm{A}(M A O A)$ genotype and high alcohol drinking have been reported in human and non-human primates. Environmental influences likely moderate genetic susceptibility. The biological basis for this interplay remains elusive, and inconsistencies call for translational studies in which conditions can be controlled and brain tissue is accessible. The present study investigated whether early life stress and subsequent adult episodic alcohol consumption affect Maoa expression in stress- and reward-related brain regions in the rat. Outbred Wistar rats were exposed to rearing conditions associated with stress (prolonged maternal separation) or no stress during early life, and given free choice between alcohol and/or water in adulthood. Transcript levels of Maoa were assessed in the ventral tegmental area, nucleus accumbens (NAc), medial prefrontal cortex, cingulate cortex, amygdala and dorsal striatum (DS). Blood was collected to assess corticosterone levels. After alcohol consumption, lower blood corticosterone and Maoa expression in the NAc and DS were found in rats exposed to early life stress compared with control rats. An interaction between early life stress and voluntary alcohol intake was found in the NAc. Alcohol intake before death correlated negatively with Maoa expression in DS in high alcohol-drinking rats exposed to early life stress. Maoa expression is sensitive to adulthood voluntary alcohol consumption in the presence of early life stress in outbred rats. These findings add knowledge of the molecular basis of the previously reported associations between early life stress, MAOA and susceptibility to alcohol misuse.

Translational Psychiatry (2015) 5, e690; doi:10.1038/tp.2015.186; published online 8 December 2015

\section{INTRODUCTION}

Globally, alcohol misuse is a major problem with health, economic and social consequences. ${ }^{1}$ Alcohol is frequently consumed for its pleasurable and euphoric effects, that is, positive reinforcing effects, but also for its sedative and stress relieving effects, that is, negative reinforcing effects. ${ }^{2,3}$ The progression from controlled drinking to compulsive drinking is crucial for the development of alcohol use disorder (AUD) and involves several neuronal circuits. ${ }^{3}$ Excessive alcohol consumption induces transcriptional changes within different mesocorticolimbic brain regions participating in stress and reward regulation, alcohol intoxication, reinforcement and addiction. ${ }^{4-6}$ The reinforcing effects of alcohol (both positive and negative) are mediated by dopaminergic neurons that originates in the ventral tegmental area (VTA) and projects to the nucleus accumbens (NAC) and prefrontal cortex, ${ }^{7}$ as well as to the cingulate cortex (CCx), dorsal striatum (DS) and amygdala $(\mathrm{Amg}){ }_{1}^{8}$ altogether forming the mesocorticolimbic pathway. In addition, a regulatory role is played by serotonergic neurons in this pathway in mediating the rewarding and reinforcing effects of alcohol, ${ }^{9,10}$ as well as by noradrenergic neurons in mediating the effects of negative emotions that arises due to withdrawal of alcohol and stress-related relapse. ${ }^{11}$ Monoaminergic neurotransmitters thus play a key role in mediating both the acute and chronic effects of alcohol. A meta-analysis, assessing alcoholinduced acute effects in rats on the neurochemistry of forebrain regions involved in neuro-circuits of AUD, reported an overall enhancement of monoaminergic transmission. ${ }^{12}$ In contrast, chronic alcohol consumption seems to have varying and region specific effects on dopaminergic neurotransmission, ${ }^{13-16}$ while serotonergic $\mathrm{c}^{9,10,16}$ and noradrenergic central neurotransmission is decreased. ${ }^{16}$ Moreover, long-term alcohol exposure induces changes in expression of monoaminergic genes in mesocorticolimbic brain regions of rats. ${ }^{17,18}$

Functional variations in key brain circuits involved in reward are likely to influence an individual's vulnerability to develop AUD. ${ }^{19}$ Individual differences in the transition into compulsive drinking and proneness to AUD depend on genetic heterogeneity and polygenicity, but also on gene by environment interactions. ${ }^{20}$ Clinical and pre-clinical studies indicate that early life stress is a risk factor for AUD later in life. ${ }^{21,22}$ One's early experience, particularly during early life, such as childhood maltreatment in humans and maternal separation (MS) in rodents, can influence the expression of genes involved in neurotransmitter systems that regulate stress response and behavior, ${ }^{23}$ and result in dysfunction of mesocorticolimbic brain pathways. ${ }^{24-26}$ This in turn can lead to stress-related disorders ${ }^{27}$ and alcohol-seeking behavior. ${ }^{28} \mathrm{How}^{-}$ ever ELS does not contribute to the development of AUD alone, but in an interaction with genetic factors. ${ }^{29,30}$

Growing evidence suggests that the monoamine oxidase $A$ (MAOA) gene, which codes for MAOA, plays an important role in regulating behavior and is linked with impulsivity, stress-related

\footnotetext{
${ }^{1}$ Department of Neuroscience, Uppsala University, Uppsala, Sweden; ${ }^{2}$ Department of Pharmaceutical Biosciences, Uppsala University, Uppsala, Sweden and ${ }^{3}$ Centre for Clinical Research, Uppsala University, County Hospital, Västerås, Sweden. Correspondence: Dr E Comasco, Department of Neuroscience, Uppsala University, Husargatan 3, BMC, Box 593, Uppsala 751 24, Sweden or Professor I Nylander, Department of Pharmaceutical Biosciences, Uppsala University, BMC, Box 591, Uppsala SE-751 24, Sweden.

E-mail: erika.comasco@neuro.uu.se or ingrid.nylander@farmbio.uu.se
}

Received 16 July 2015; revised 28 September 2015; accepted 21 October 2015 
disorders and AUD. $^{31}$ Monoamine neurotransmitters such as serotonin, dopamine and norepinephrine, that are mediating the effects of alcohol and are crucial for reward and initiation of alcohol reinforcement, ${ }^{7}$ are metabolized by MAOA in humans ${ }^{31}$ as well as in rats (Maoa). ${ }^{32}$ In 1993, mutations in the MAOA gene, resulting in deficiency of $M A O A$, were identified in human males presenting abnormal behavior such as impulsive aggression, arson, and attempted rape. ${ }^{33}$ Later, a functional polymorphism was discovered in the promoter region of the MAOA gene (MAOAuVNTR) in humans and shown to affect the transcriptional activity in transfected non-neuronal cells, with the 3.5 or 4-repeat allele having 2-10 times higher activity than the 3 or 5-repeat allele. ${ }^{34,35}$ Whereas, in vivo studies do not find any association between MAOA-uVNTR genotype and its transcriptional activity, in human $^{36,37}$ as well as non-human primates. ${ }^{38}$ Several studies reported an association of both the high and low activity MAOAUVNTR alleles with antisocial behavior, ${ }^{39-41}$ and susceptibility to AUD, ${ }^{42,43}$ while others found an association with alcohol misuse but only in an interaction with stressful life events. ${ }^{44-47}$ On the contrary, some other studies found no association between MAOA-UVNTR and AUD. ${ }^{48,49}$ Furthermore studies of primates provide a somewhat discordant evidence. Mother-reared male rhesus monkeys carrying the low activity rhMAOA-uVNTR allele display higher aggression scores as adults, ${ }^{50}$ whereas no effect was found in adolescent and young adult males in one study, ${ }^{38}$ while in another study the ones carrying the high activity rhMAOAuVNTR allele consumed higher amount of alcohol than those carrying the low activity allele. ${ }^{51}$ Altogether, these studies suggest $M A O A$ as a candidate gene sensitive to gene by environment interactions and associated with AUD. However, they also call for translational studies to clarify the inconsistencies in MAOA-byenvironment interaction effects ${ }^{52-54}$ and deepen knowledge on potential molecular mechanisms behind this interaction allowing accessibility of the brain. In fact, though several studies have attempted to investigate $M A O A$ genotype by environment interaction and its effect on behavior and AUD, the connecting link, that is the biological basis behind such interaction, is missing. Hence, the hypothesis that early life stress and alcohol consumption, alone or in combination, would be associated with altered expression of Maoa in brain areas related to stress-regulation and reward processing in adult rats was tested in the present study.

The aim was to assess whether early life stress in the form of prolonged MS, and adult episodic voluntary alcohol consumption, affect Maoa expression in brain regions related to stress and reward in outbred rats. First, the effects of voluntary alcohol drinking and the potential confounder of single housing were investigated in rats subjected to conventional animal facility rearing conditions. Second, the long-term effect of early life stress was examined using a rodent model where rats were exposed to prolonged and brief, respectively, MS during the first three postnatal weeks ${ }^{55,56}$ and to voluntary episodic alcohol drinking in adulthood. Finally, the interaction and main effect of prolonged MS and alcohol intake were investigated. Correlations between Maoa expression in different regions were tested in each experimental group, as well as between Maoa and alcohol intake in the experimental groups exposed to alcohol.

\section{MATERIALS AND METHODS}

\section{Animals}

Time-mated Wistar dams ( $n=25$; RccHan:WI, Harlan Laboratories, Horst, The Netherlands) arrived at gestation day 15. After birth (post-natal day (PND) 0), the pups were sexed and cross-fostered to avoid the use of biological littermates in the same experimental groups. Each litter was arranged to 10 pups, 6 males and 4 females, and the litters were randomly assigned to the different experimental groups. Only males were used in the present study. The study was performed all in one, the researcher was not blind to the animal experiment, however, genetic and hormone analyses were performed in a blind manner. The study was approved by the Uppsala Animal Ethical Committee (C32/11) and followed the guidelines of the Swedish Legislation on Animal Experimentation (Animal Welfare Act SFS1998:56) and the European Communities Council Directive (86/609/EEC).

\section{Early life rearing conditions}

A schematic outline of the experimental design in the study is illustrated in Figure 1. A rodent MS model was used to simulate different early life conditions during the first 3 post-natal weeks. Based on previous studies, prolonged daily MS (360 min; MS360) was used to simulate a risk environment ${ }^{56}$ and short MS (15 min; MS15) was used as a control to MS360. The separations were performed during the light period and started at 0900 hours. The MS procedure has been described in detail elsewhere. ${ }^{57}$ The litters were weighed on PND $0,3,7,10,13,16$ and the cages were changed on PND 7 and 16. The separations were always performed in the same animal rooms and only one person performed all separation and care taking. Animal facility reared (AFR) rats were included in the study for assessment of single housing and alcohol drinking in rats subjected to conventional laboratory rearing conditions. The animals in the AFR group were left undisturbed with the exception of cage change (PND 7,16 ) and weighing of the litter (PND 0, 7, 16). On PND 22, all animals were weaned and the light/dark cycle was switched to lights off between 0600 to 1800 hours until the end of the experiment. The rats were grouphoused, three per cage, during adolescence (Figure 1a).
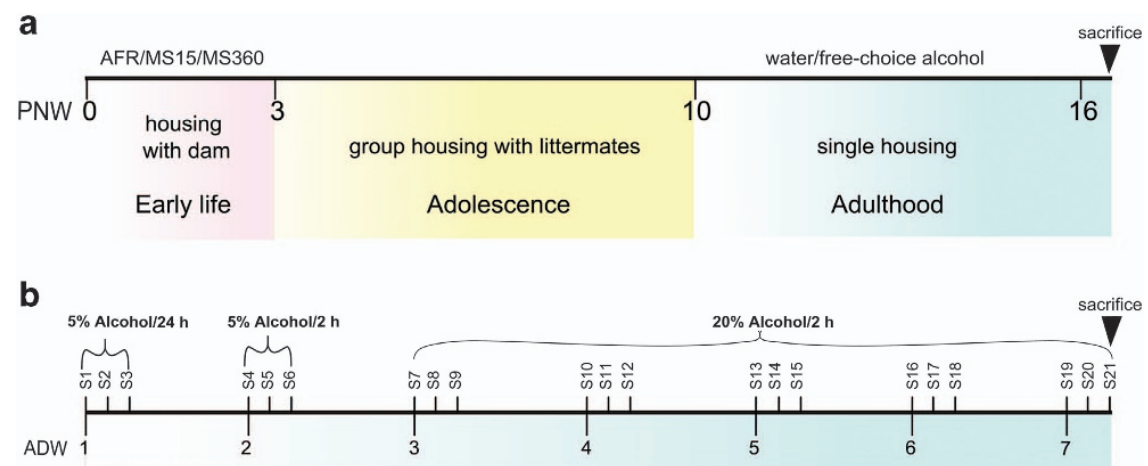

Figure 1. Experimental design from post-natal weeks (PNW) 0 to 16. (a) Male Wistar rats were subjected to MS15, MS360 or AFR the first three post-natal weeks and exposed to voluntary alcohol consumption from PNW 10 to 16 . All animals, except one group of AFR rats ( $n=9$ ), were single-housed from PNW 10. (b) Detailed scheme of the voluntary drinking paradigm. A two-bottle choice paradigm was used with free access to either alcohol/water or water/water for 3 consecutive days per week from PNW 10 to 16. The access to alcohol was limited to 2-h sessions. The rats were killed during ADW 7, immediately after a 2-h drinking session. ADW, alcohol-drinking week; AFR, animal facility reared; MS15, maternal separation for 15 min, MS360, maternal separation for 360 min; PNW: post-natal week; S, session. 
Voluntary alcohol consumption

On post-natal week (PNW) 10 the MS rats were randomly assigned to water-drinking (MS15W, $n=10$; MS360W, $n=10$ ) and alcohol-drinking groups (MS15A, $n=10 ;$ MS360A, $n=20$ ). More rats were included in the MS360 group based on previous findings of subgroups with responder and non-responder rats regarding the MS effect on alcohol intake. ${ }^{56}$ The AFR rats were also assigned to water (AFRW, $n=9$ ) and alcohol drinking (AFRA, $n=11$ ), respectively. All rats were single-housed for individual fluid measurements until decapitation at PNW 16. One additional group of AFR rats $(n=7)$ was group housed during PNW 10 to 16 . The rats had free choice between non-sweetened alcohol (5 or $20 \%$ made from ethanol 96\%; Solveco, Rosersberg, Sweden) and water during the dark cycle. Water-drinking controls had two bottles with water.

A detailed scheme of the voluntary alcohol exposure paradigm is shown in Figure 1b. During the first alcohol-drinking week (ADW), the rats had free access to $5 \%$ alcohol for $24 \mathrm{~h}$ and the next week limited access to $5 \%$ for $2 \mathrm{~h}$ for 3 consecutive days a week; the following 5 weeks they had access to $20 \%$ alcohol in 2-h sessions for 3 consecutive days a week followed by 4 days of no alcohol with free access to water (Figure 1b). Alcohol and water were changed every session and the bottle position was altered every day to avoid position preference. Bottles with nipples were used to avoid spillage biases and at the end of each session the alcohol and water intake was quantified by weighing the bottles. The individual drinking patterns over time were assessed from ADW 1 to 7 . At post-natal week 16, that is, ADW 7, all rats were killed. The animals were brought to a separate room for decapitation immediately after the drinking session 21 (Figure 1b). To assess correlations between individual drinking behavior and Maoa expression in different brain regions, the average alcohol intake during ADW 6 and ADW 7 were considered; ADW 6 because it is the drinking last week without any putative disturbances due to persons entering the room and more representative of the individual drinking pattern; and ADW 7 because it reflects the effect of most recent alcohol intake at the time-point of death which is more relevant in terms of alcohol-induced effects on gene expression. The VTA, NAc, medial prefrontal cortex (mPFC), CCx, Amg and DS were removed from the brain and immediately frozen on dry ice and stored at $-80^{\circ} \mathrm{C}$. At the same time, trunk blood was collected and stored at $-80^{\circ} \mathrm{C}$ for studying blood corticosterone levels.

\section{Gene expression analyses}

RNA isolation. RNA was isolated from rat VTA, NAc, mPFC, CCx, Amg and DS using AllPrep DNA/RNA/miRNA Universal Kit according to the manufacturer's protocol (Qiagen, Sollentuna, Sweden). Quantification of the nucleic acid was carried out using a Nanodrop ND 1000 spectrometer (Thermo Fisher Scientific, Wilmington, DE, USA).

CDNA synthesis. RNA (700ng) was converted to complementary DNA (cDNA) using the QuantiTect Reverse Transcription Kit (Qiagen). The manufacturer's protocol was followed including a genomic DNA wipe-out reaction. The final $\mathrm{CDNA}$ synthesis reaction was performed at $42^{\circ} \mathrm{C}$ for $35 \mathrm{~min}$, and the reaction was inactivated at $95^{\circ} \mathrm{C}$ for $5 \mathrm{~min}$. The newly synthesized CDNA was diluted 20 times with distilled water and stored at $-20{ }^{\circ} \mathrm{C}$ until further analysis.

qPCR analyses. Diluted CDNA $(20 \times)$ was used to assess the expression of Maoa, as well as Actb, Gapdh and Rpl19, as housekeeping genes, using CFX96 Touch Real-Time PCR Detection System real-time PCR (Applied Biosystems, Foster City, CA, USA). Primers were designed using Primer 3 (http://frodo.wi.mit.edu/) and cross-checked using Primer Map (http:// www.bioinformatics.org/sms2/primer map.html) (Supplementary Table S1). The final reaction mixture of $20 \mu \mathrm{l}$ contained iQ SYBR Green Supermix (Bio-Rad Laboratories, Hercules, CA, USA), $0.15 \mu \mathrm{M}$ of each primer and $3 \mu \mathrm{l}$ cDNA template; and each sample was run in triplicates. The PCR cycling conditions were as follows: hot start at $95^{\circ} \mathrm{C}$ for $3 \mathrm{~min}$; denaturation at $95^{\circ} \mathrm{C}$ for $10 \mathrm{~s}$; annealing for $30 \mathrm{~s}$ and extension at $72{ }^{\circ} \mathrm{C}$ for $45 \mathrm{~s}$ (Supplementary Table S1). Cycle was repeated 40 times. A three-step control was performed to assess genomic DNA contamination: (1) On column DNase treatment during the extraction process; (2) genomic DNA wipe-out reaction prior to CDNA synthesis; (3) Designing of primers across two adjacent exons to avoid any unspecific amplification of genomic DNA. Moreover, each real-time PCR plate contained samples belonging to all experimental groups. Each plate contained a positive and a negative control sample. Gel electrophoresis images illustrating the primer specificity of the genes-of-interest and the RNA integrity of $10 \%$ of randomly selected samples from the regions-of-interest are presented in the Supplementary Figure S1.

Data analysis. Data of the relative fluorescence unit were collected using Biorad CFX manager 3.1 software (Applied Biosystems). Cq values were computed using the LinregPCR open source software, ${ }^{58}$ and normalized adjusting for plate bias. Corrected $\mathrm{Cq}$ that had a s.d. $>0.5$ were excluded. Relative gene expression levels were determined using the $\triangle C T$ method (Biorad real-time PCR application guide, Bio-Rad, \#170-9799). Three housekeeping genes (Actb, Gapdh and Rp/19) were used to calculate the relative gene expression for all the brain regions-of-interest, except VTA and DS. For VTA, Actb was excluded because the negative control displayed amplification, whereas for DS Gapdh was excluded because $\mathrm{Cq}$ values were found to be significantly different between groups. All the laboratory and preprocessing analyses were performed in a blind manner.

\section{Corticosterone analyses}

Samples were analyzed using the commercial ImmuChem Double Antibody Corticosterone $125^{1}$ RIA kit for rats and mice (MP Biomedicals, Orangeburg, NY, USA) in accordance with the included protocol, with the exception of the addition of one standard $\left(12.5 \mathrm{ng} \mathrm{ml}^{-1}\right)$. All samples were analyzed in duplicate. According to the protocol in the RIA kit, the intraassay variation was $4.4-10.3 \%$ and the inter-assay variation $6.5-7.2 \%$. The corticosterone antiserum showed $100 \%$ cross-reactivity with corticosterone, while cross-reactivity to other steroids was $0.34 \%$ to deoxycorticosterone, $0.10 \%$ to testosterone, $0.05 \%$ to cortisol and $<0.05 \%$ to other tested steroids. Blood corticosterone levels are expressed in terms of $\mathrm{ng} \mathrm{ml}^{-1}$.

\section{Statistics}

Gene expression data were not normally distributed, except for DS $(P=0.809)$, as assessed by Shapiro-Wilk's test, therefore the non-parametric Kruskal-Wallis and Mann-Whitney tests were performed to compare the relative gene expression, corticosterone levels and alcohol intake between three and two experimental groups, respectively. Significance was reported as exact Sig. $[2 \times$ (one-tailed Sig.)]. Bivariate correlations were assessed using the non-parametric Spearman test with significance reported as two-tailed significance. To test for correlations between Maoa expression in different brain regions and alcohol intake, data of alcohol intake during ADW 6 and 7 were considered. There was homogeneity of variances in gene expression data between groups, as assessed by Levene's non-parametric test for equality of variances $(P>0.05)$, therefore the general linear model based on two-way ANOVA, mean of squares type III, was performed to investigate main and interaction effects of MS and alcohol. Whisker box plots were used for illustrative purposes. All statistical analyses were performed using the IBM SPSS software (version 22, IBM, Armonk, NY, USA) and significance was set at $P \leqslant 0.05$.

\section{RESULTS}

Maoa expression levels in all brain regions-of-interest are shown for each experimental group in Supplementary Table S2.

Effect of housing and alcohol consumption on Maoa expression in AFR rats

Single housing in adult rats had no effect on Maoa expression as indicated by no significant differences between single- and grouphoused AFR rats in the VTA, NAc, MPFC, CCx, Amg and DS. Furthermore, there were no significant differences between waterand alcohol-drinking AFR rats, that is, 7 weeks of voluntary drinking had no effect on Maoa expression in rats reared in a conventional laboratory setting. The voluntary alcohol consumption in the AFR rats is shown in Supplementary Table S3A.

\section{Effect of prolonged MS and alcohol consumption on Maoa expression in MS rats}

The voluntary alcohol consumption in adult MS15 and MS360 rats is shown in Supplementary Table S3A. Alcohol drinking affected Maoa expression in the NAC and DS, whereas no differences were found in the VTA, mPFC, CCx and Amg. In the NAc, an interaction 

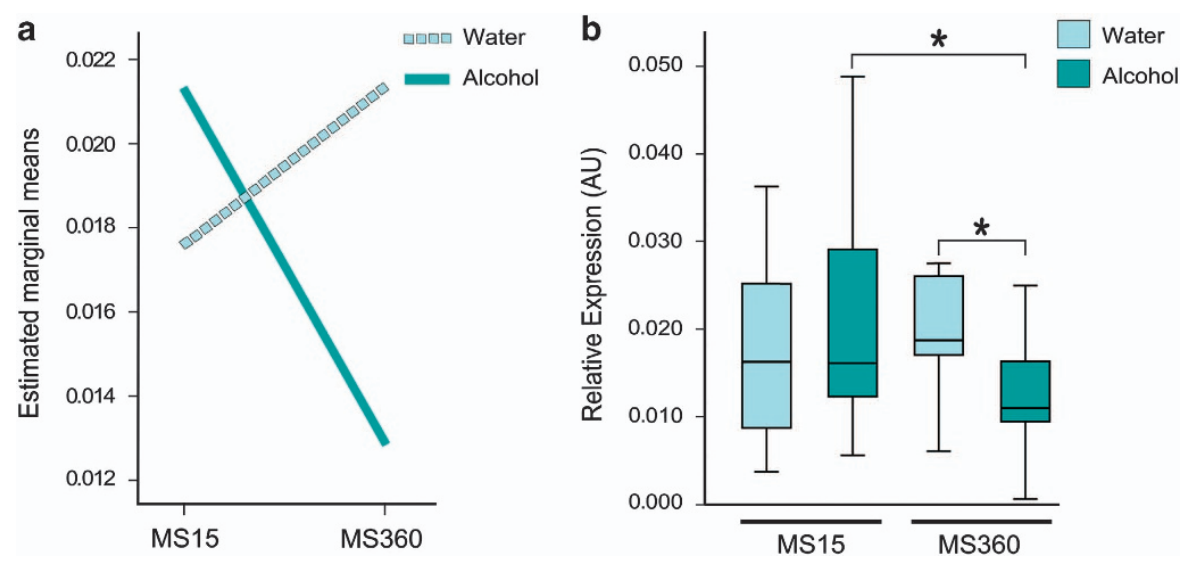

Figure 2. Maoa expression in NAc of rats exposed to short or prolonged MS and water or free choice alcohol. (a) Interaction effect of stress and alcohol on Maoa expression in the NAc in MS15 and MS360 rats ( $\mathrm{F}=3.949 ;$ Adj. $\left.R^{2}=0.07 ; P=0.05\right)$; (b) Lower Maoa expression in the NAc of voluntary alcohol-drinking rats exposed to MS360 compared with water-drinking rats $(U=52 ; * P=0.05)$ and MS15 alcohol-drinking rats $(U=52$; $P=0.05)$. MS15, maternal separation for $15 \mathrm{~min}$; MS360, maternal separation for $360 \mathrm{~min}$; NAc, nucleus accumbens.

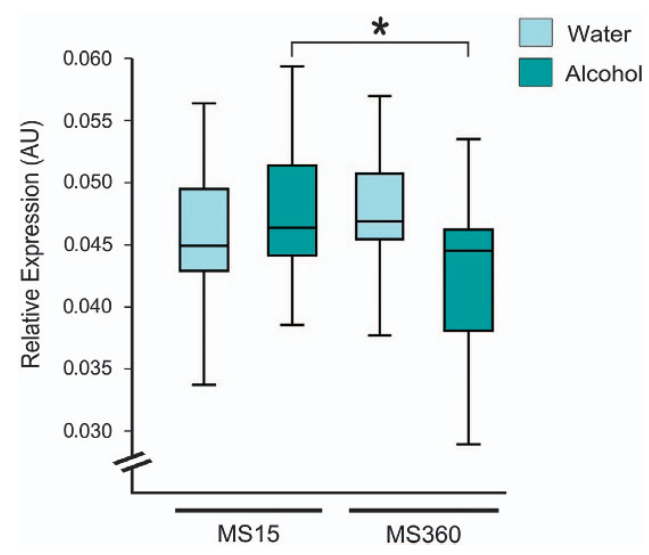

Figure 3. Maoa expression in the DS of rats exposed to short or prolonged MS and water or free choice alcohol. Lower Maoa expression in the DS of voluntary alcohol-drinking rats exposed to MS360 compared with alcohol-drinking MS15 rats $(U=55$; $\left.{ }^{*} P=0.049\right)$. DS, dorsal striatum; MS, maternal separation.

effect between MS and alcohol was present (Figure 2a). Alcohol drinking MS360 rats had lower Maoa expression compared with water-drinking MS360 rats, whereas no difference was observed between water- and alcohol-drinking MS15 rats (Figure 2b). Furthermore, alcohol-drinking MS360 rats also had lower Maoa expression compared with alcohol-drinking MS15 rats (Figure 2b). In the DS, lower Maoa expression was found in voluntary alcohol-drinking rats exposed to MS360 compared with MS15 alcohol-drinking rats, whereas no difference was found in comparison to MS360 water-drinking rats (Figure 3). No main or interaction effect of early life stress or alcohol consumption was found in VTA, MPFC, CCX and Amg.

Correlation between Maoa expression and alcohol intake in MS360As rats

As expected, ${ }^{57,59}$ the majority of the rats that reached a high voluntary consumption ( $>1.5 \mathrm{~g} \mathrm{~kg}^{-1}$ per $2 \mathrm{~h}$ ) during the ADW 6 (S16 to S18) and ADW 7 (S19 to S21) were MS360 rats (Supplementary Table S3A, Supplementary Figure S2). To assess correlations between individual drinking behavior and Maoa expression in different brain regions, the average alcohol intake for ADW 6 and ADW 7 were considered (see materials and methods). Rats exposed to MS360 were sub-grouped into low ( $\leqslant$ $1.5 \mathrm{~g} \mathrm{~kg}^{-1}$ per $2 \mathrm{~h}$ ) and high (>1.5 $\mathrm{g} \mathrm{kg}^{-1}$ per $2 \mathrm{~h}$ ) alcoholdrinking rats according to ADW 6 (Supplementary Table S3B) and ADW 7 (Supplementary Table S3C). According to ADW 6 subgrouping, no correlation was found between expression in any of the brain region-of-interest and alcohol intake at ADW 6 in MS360 high as well as low alcohol-drinking rats. While according to ADW 7 subgroupings, a strong negative correlation was found between alcohol intake at ADW 7 and expression in DS in MS360 high alcohol-drinking rats $(r=-0.943, P=0.005)$. In MS360 low alcoholdrinking rats, no correlation was found between expression in any of the brain region-of-interest and alcohol intake at ADW 7. Also, Maoa expression was not significantly different in high compared with low alcohol-drinking MS360 rats depending on both ADW 6 and 7 , in any of the brain region-of-interest.

Correlation of Maoa expression between brain regions-of-interest in each experimental group

Group-wise correlations were found between Maoa expression in different brain regions, mainly between NAc and MPFC (Table 1). In all groups, except the rats exposed to early life stress and alcohol (MS360As), Maoa expression in NAc and mPFC was positively correlated. In addition, scattered and group-dependent correlations were also observed between the other regions (Table 1).

Effect of prolonged MS and alcohol consumption on corticosterone levels in MS rats

Alcohol drinking MS360 rats displayed lower corticosterone levels compared with alcohol-drinking MS15 $(U=22 ; P=0.033)$, and water-drinking MS360 rats $(U=20 ; P=0.003)$ (Figure 4). While between water- and alcohol-drinking MS15 rats, no significant difference was found in the corticosterone levels, likewise between MS15 and MS360 water-drinking rats.

Group-wise correlations were studied between blood corticosterone levels and Maoa expression in different brain regions (VTA, NAc, mPFC, CCx, Amg and DS). No correlation was found in water- or alcohol-drinking MS360 rats, but a positive correlation in Amg in water-drinking MS15 rats $(r=0.842, P=0.002)$. 
Table 1. Group-wise correlations of Maoa expression in different brain regions-of-interest ${ }^{\mathrm{a}}$

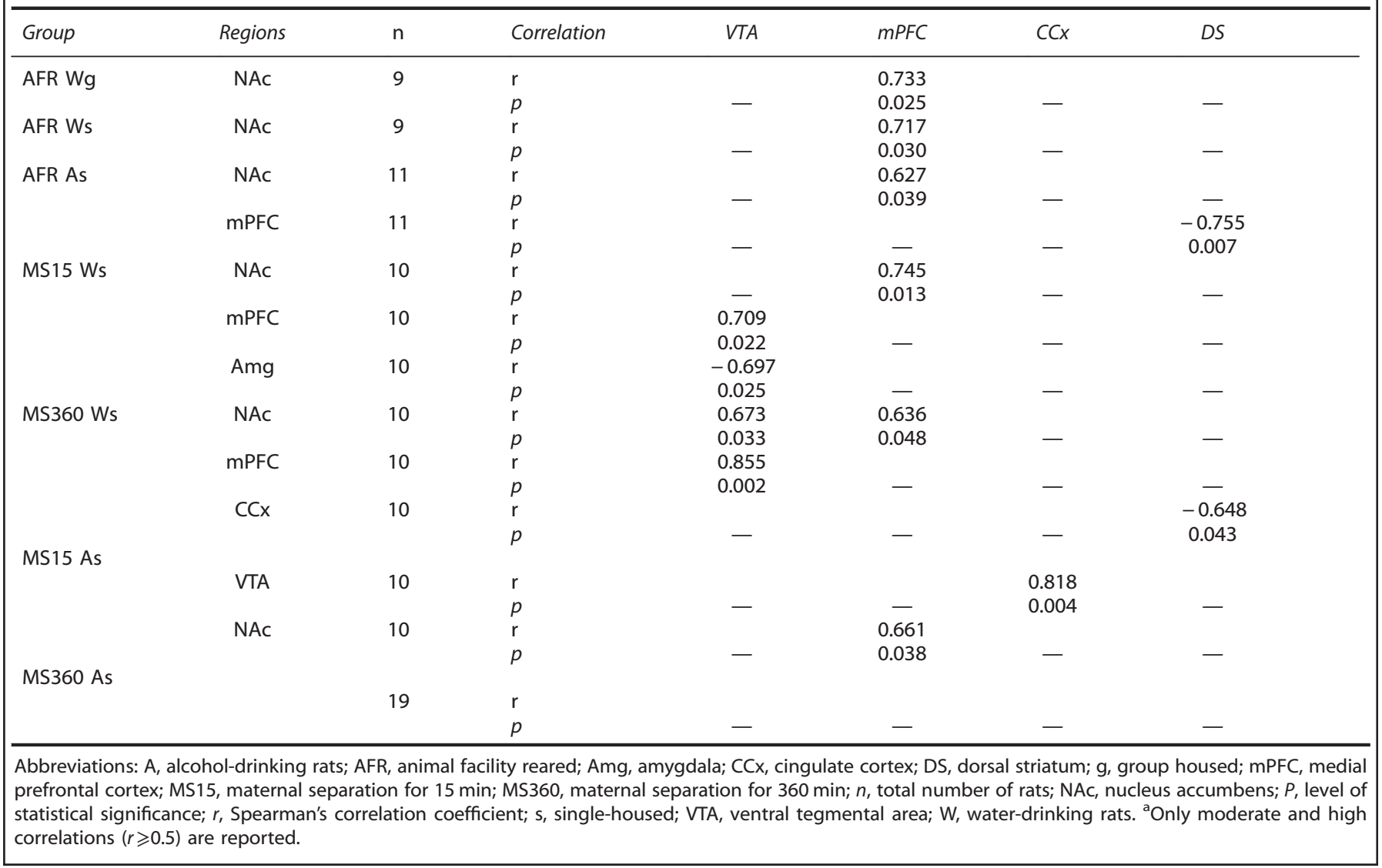

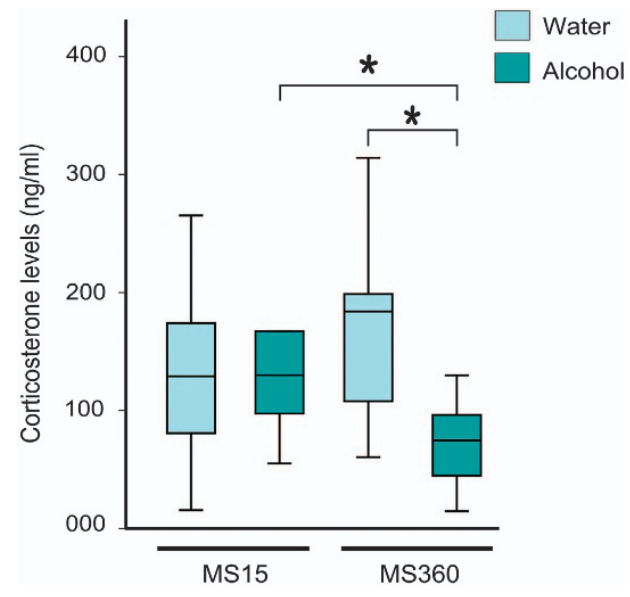

Figure 4. Blood corticosterone levels in MS rats. Lower blood corticosterone levels in MS360 voluntary alcohol-drinking rats compared with alcohol-drinking MS15 rats $\left(U=22 ;{ }^{*} P=0.033\right)$, as well as compared with water-drinking MS360 rats $(U=20$; $\left.{ }^{*} P=0.003\right)$. MS15, maternal separation for $15 \mathrm{~min}$; MS360, maternal separation for $360 \mathrm{~min}$.

\section{DISCUSSION}

Early life stress, voluntary alcohol consumption and Maoa expression

The effect of voluntary episodic alcohol consumption on Maoa expression was examined in stress- and reward-related key brain regions in young adults outbred male Wistar rats subjected to early life stress (MS360) or no stress. Lower Maoa expression was found in NAC and DS of ethanol drinking MS360 rats. These results are in keeping with human studies where a MAOA-uVNTR genotype conferring low transcriptional activity was more common in adolescent males, who had poor family relationship or had been sexually abused or maltreated in childhood, and selfreported higher alcohol consumption. ${ }^{44,47}$ The present findings suggest a heightened sensitivity of the NAc and DS monoaminergic systems of rats with a background of early life stress to the effects of alcohol, compared with rats without a stressful background.

Monoamines are expressed pre- and post-synaptically during early post-natal development, and have a critical role in modulation of neurodevelopment of functional limbic circuits that allows adaptations to environmental factors. ${ }^{26}$ MAOA is indeed strongly expressed during embryonic life in all serotoninergic neurons progressively declining from PND 0 to 10, in adrenergic neurons and post-natally in most transient and permanent dopaminergic neurons. ${ }^{60}$ Maoa knock-out mice ${ }^{61}$ and transgenic Maoa-deficient mice ${ }^{62}$ are maximally affected during early post-natal life displaying higher levels of monoamines, and stress-related phenotypes including increased aggression in adulthood. Thus, considering the role of Maoa in developmental processes shaping adult behavior, ${ }^{26}$ and that early life stress impacted in the present study during a sensitive period of monoaminergic development, it is likely that MS360 rats have a Maoa-related sensitivity to alcohol linked to early life stress. It could be hypothesized that ELS would alter Maoa expression in other stress-related regions, such as the locus coeruleus and raphe nuclei, of voluntary alcohol-drinking MS360 rats; however, these regions were not available for investigation in the present study.

Maoa expression in NAc and mPFC was positively correlated in all groups, except in alcohol-drinking MS360 rats. 
A straightforward functional interaction exists between mPFC and NAc via dopaminergic neurotransmission in such a way that they are involved with opposite effects on reward mechanisms. ${ }^{63}$ Depletion of dopaminergic activity in MPFC enhances stress as well as reward induced dopamine release in NAc hence acting as some kind of negative feedback, and disruption of this inhibitory control increases the vulnerability to alcohol misuse. ${ }^{63}$ The present correlative pattern points to a disrupted monoaminergic functional interaction of NAc with mPFC following exposure to both early life stress and subsequent consumption of alcohol. Thus, brain region- as well as network-based alterations of MAOA likely underpin early life stress-mediated propensity to alcohol consumption.

Elevated brain MAOA expression and blood corticosterone levels are a result of chronic stress and putative risk factors for psychiatric disorders. ${ }^{64}$ Corticosterone levels were higher in water compared with alcohol-drinking MS360 rats, as well as Maoa expression in NAc and tendentially in DS, indicating signs of stress. Nevertheless, in rats exposed to early life stress no correlation was found between blood corticosterone and Maoa expression, neither in water nor alcohol-drinking rats, in any brain region. Yet, a previous study, employing peri-pubertal stress, reported higher blood corticosterone levels and fronto-cortical Maoa expression in stressed adult male rats. ${ }^{65}$ In the present study, after consumption of alcohol, MS360 rats displayed lower corticosterone levels and Maoa expression. Hence MS360 rats likely consumed alcohol for stress relieving purpose, which in turn alleviated stress, as illustrated by lower corticosterone and Maoa expression in these rats.

\section{Voluntary alcohol consumption and Maoa expression}

Lower Maoa expression in NAc and DS presumably leads to decreased metabolism and subsequently increased synaptic concentration of monoamines, accompanied by an increased rewarding response to alcohol. Alcohol intake during the 7 weeks of voluntary consumption increased over time in rats exposed to prolonged MS. As expected from earlier studies, ${ }^{56,57,59}$ subgroups were observed with some MS360 rats acquiring higher consumption throughout the drinking period. When investigating Maoa expression in MS360 rats that acquired a high alcohol intake at ADW 6 and 7, a strong negative correlation was observed between alcohol intake at ADW 7 and Maoa expression in DS; whereas no significant difference was found in any of the brain regions-ofinterest comparing MS360 rats with high vs low alcohol intake. Similarly, no difference in MAOA activity has been found in whole brain homogenate from alcohol compared with non-alcoholpreferring rats, ${ }^{66}$ in the brainstem, cerebellum, cortex, hippocampus and striatum of rats exposed to alcohol for 90 weeks, ${ }^{67}$ and in the whole brain of rats exposed to both voluntary alcohol intake ( $1 \mathrm{~h}$ per day, 34 weeks) as well as alcohol vapors $(5 \mathrm{~h}$ per day, 7 weeks). ${ }^{68}$ While upregulation of Maoa in CCx of Wistar rats has been reported 3 weeks after termination of long-term exposure to ethanol vapors, NAc was not investigated. ${ }^{17}$ Also, a meta-analysis reported enhanced monoamines levels in forebrain regions of rats after acute alcohol exposure. ${ }^{12}$ In humans, MAOA activity was found to be lower in the hypothalamus and caudate nucleus of chronic alcoholics compared with healthy subjects. ${ }^{66}$ Moreover, low MAOA expression in blood and in the core of the NAC associated with high chronic voluntary alcohol consumption in male non-human primates. ${ }^{38}$ It is plausible that methodological differences could explain these discrepant results (for example, alcohol-drinking paradigm and duration).

Previous studies have mainly assessed levels of monoamines (that is, 5-HT, DA and NE) and their major metabolites (that is, 5-hydroxyindoleaceticacid (5-HIAA), homovanillic acid and 3-methoxy-4-hydroxyphenylglycol (MHPG), respectively) in cerebrospinal fluid ${ }^{48,69,70}$ or brain tissue, ${ }^{71,72}$ to investigate alterations in monoamine function and metabolism in the brain of high alcohol-drinking rats or alcoholics. Rats selectively bred for high alcohol drinking were reported having lower levels of 5-HT and 5-HIAA in the hypothalamus, cerebral cortex, DS, NAc, and lower levels of DA and 3,4-dihydroxyphenylacetic acid and homovanillic acid in the NAc and anterior striatum, compared with low alcohol-drinking rats, ${ }^{72}$ likewise in alcohol-preferring compared with non-preferring rats. ${ }^{73,74}$ Lower rate of DA metabolism and higher DA cerebrospinal fluid levels were observed in rhesus macaques after 12 months of high voluntary alcohol consumption. ${ }^{38}$ In humans, low levels of monoamine metabolites were found in the cerebrospinal fluid of alcoholic patients. ${ }^{69,70,75} \mathrm{~A}$ study of Finnish male criminal alcoholics found lower levels of homovanillic acid and MHPG compared with control but no difference observed in 5-HIAA levels, and a trend of association between the low activity MAOA-uVNTR with higher levels of homovanillic acid, 5-HIAA metabolite levels in both alcoholics as well as healthy subjects. ${ }^{48}$ Altogether, these studies provide corroborative evidence of an alcohol-driven downregulation of monoaminergic neurotransmission, though the underlying mechanisms remain unknown.

Rats lack the MAOA-uVNTR polymorphism, on the contrary to human and non-human primates. Some, but not all, ${ }^{48,49}$ human studies report an association between the low transcriptional activity allele of MAOA-uVNTR and higher risk of AUD. ${ }^{42,43}$ In addition, in non-human primates the high transcriptional activity allele of rhMAOA-uVNTR was found to be associated with high alcohol consumption, ${ }^{51}$ or no association was found. ${ }^{38}$ Discrepancies in genetic association studies possibly reflect the contradictory in vitro, ${ }^{34,35}$ relative to in vivo, ${ }^{36-38}$ findings about the functionality of this polymorphism. In addition, they make room to speculate on early-in-time susceptibility, which in turn might be smoothed over time by epigenetic mechanisms in a favorable or disadvantageous manner. In fact, low blood MAOA expression, but not rhMAOA-uVNTR genotype, predicts high voluntary alcohol consumption in rhesus macaques. ${ }^{38}$ Taken together with the present findings, there is suggestive evidence that both the transient decrease in Maoa expression following alcohol exposure as well as low levels of MAOA throughout life time (presumably due to genetic variation in the MAOA) would predict risky alcohol consumption behavior in adulthood.

\section{Methodological strengths and limitations}

Wistar pups were subjected to early life stress in a rodent model showing good face and construct validity in studies of early life impact on adult voluntary alcohol consumption. ${ }^{55,56}$ Prolonged maternal separation (MS360) every day for the first 3 PNWs simulates early life stress through disturbed social interactions, and is a risk environment for high adult alcohol consumption whereas MS15 is associated with low alcohol intake. ${ }^{56,76}$ Besides providing a protective environment, MS15 serves as control for MS360 since rats in both groups are exposed to the same handling procedures.

In adulthood, rats were single-housed and given a two-bottle free choice between water and alcohol with intermittent access to alcohol ( 3 consecutive days followed by 4 days of no alcohol with only access to water) to mimic episodic alcohol drinking in humans, ${ }^{77}$ for a limited period $(2 \mathrm{~h}),{ }^{78,79}$ for 7 successive weeks. Studies suggest that intermittent and limited access to alcohol induces higher voluntary alcohol intake compared with continuous access for $24 \mathrm{~h}^{80-83}$ and have the advantage of producing neurochemical adaptations that are relevant to AUD. ${ }^{84}$

The MS360 rats were sub-grouped into high $\left(>1.5 \mathrm{~g} \mathrm{~kg}^{-1}\right.$ per $2 \mathrm{~h}$ ) and low ( $\leqslant 1.5 \mathrm{~g} \mathrm{~kg}^{-1}$ per $2 \mathrm{~h}$ ) alcohol-drinking groups based on previous knowledge of blood alcohol concentration (BAC) depending on amount of alcohol consumed. For example, rats at 10 weeks of age had $B A C \approx 70 \mathrm{mg} \mathrm{dl}^{-1}$ after $1.5 \mathrm{mg} \mathrm{kg}^{-1}$ alcohol 
intake, as similarly shown in adult rats having a $\mathrm{BAC} \approx 80 \mathrm{mg} \mathrm{dl}^{-1}$ $2 \mathrm{~h}$ after exposure to $2 \mathrm{~g} \mathrm{~kg}^{-1.85}$ Nevertheless, the high alcohol consumption level of $1.5 \mathrm{~g} \mathrm{~kg}^{-1}$ per $2 \mathrm{~h}$ is relative to the Wistar outbred rats used in the present study. Though the alcohol consumption is low compared with rats in the addictive state or genetically alcohol-preferring rats, the purpose of the present study was to investigate early neurobiological signatures related to episodic voluntary alcohol intake in non-addicted and non-preferring rats.

As single housing in the home cage is required in voluntary drinking paradigms, the effect of single housing on Maoa expression in adult AFR rats, that is, rats subjected to conventional rearing conditions, was studied in the present study. Single housing did not affect Maoa expression when compared with group housing, indicating no confounding effects due to housing conditions. Furthermore, voluntary alcohol drinking did not affect Maoa expression in the AFR rats in comparison to water-drinking rats hence suggesting that voluntary alcohol consumption does not affect Maoa expression in normal or non-stressful conditions (animal facility rearing, in this case).

Only male rats were herein investigated calling for studies of females. Sex differences have been implicated in human studies on MAOA-uVNTR genotype, and its interaction with early life environment, as well as in rodents regarding the impact of maternal separation on voluntary alcohol intake. ${ }^{76,86}$ Moreover, ample evidence exists of influences of the gonadal hormones on the monoaminergic mesocorticolimbic circuit functioning and the response to stressors and vulnerability to psychiatric illness. ${ }^{87-89}$

The present study tested a previously described definite hypothesis, and no correction for multiple testing was applied. Restrictions, such as the Bonferroni correction, are concerned with a general null hypothesis $\left(\mathrm{H}_{0}\right)$ and are employed to reduce type I errors, as well as reduce the statistical power to reject a false null hypothesis rendering truly important differences as non-significant since findings depend on a number of other tests performed that in turn inflate the likelihood of accepting type II errors. $^{90,91}$

\section{CONCLUSIONS}

Knowledge of the effects of voluntary alcohol drinking on Maoa expression in brain regions related to reward and addiction is limited. The present results show that voluntary drinking in adulthood was associated with low Maoa expression in the NAC and DS but only in rats previously exposed to early life stress. No differences were seen in water-drinking control rats exposed to stress or no stress, indicating that voluntary alcohol consumption is necessary to reveal a monoaminergic dysfunction in animals exposed to early life adverse conditions. These findings expand current knowledge on the molecular basis of the previously reported associations between early life stress, Maoa and susceptibility to excessive alcohol consumption and AUD. Further studies are needed to investigate mechanistic signatures behind $M A O A$-driven susceptibility to AUD. Efforts should be convened to investigate the mesocorticolimbic monoaminergic system during early life and at an early stage of alcohol-drinking behavior.

\section{CONFLICT OF INTEREST}

The authors declare no conflict of interest.

\section{ACKNOWLEDGMENTS}

The present work was partially supported by funds from the Alcohol Research Council of the Swedish Alcohol Retailing Monopoly, the European Foundation for Alcohol Research (EA 11 30), the Swedish Research Council (K2012-61X-22090-01-3) to IN; from the Swedish Council for Working Life and Social Research (2011-0627), Fredrik and Ingrid Thuring foundation (2012, 2013), Lars Hierta's Minne foundation
(2013), Swedish Brain foundation (PS2013-0052), and Lundberg's and Karlsson's foundation (2013) to EC; and the Swedish Brain Foundation, the Alcohol Research Council of the Swedish Alcohol Retailing Monopoly, the Swedish Council for Working Life and Social Research (FAS), the Uppsala and Örebro Regional Research Council, the Fredrik and Ingrid Thurings Foundation, the County Council of Västmanland, the König-Söderströmska Foundation, the Swedish Psychiatric Foundation and the Svenska Spel Research Foundation to KWN. We wish to thank Linnea Grannholm at the Department of Pharmaceutical Biosciences, as well as Aniruddha Todkar, Maria Sapounidou, Maria Vrettou and Sofia Bergman at the Department of Neuroscience, Neuropsychopharmacology group, for their contribution to the laboratory work.

\section{REFERENCES}

1 Rehm J, Mathers C, Popova S, Thavorncharoensap M, Teerawattananon Y, Patra J. Global burden of disease and injury and economic cost attributable to alcohol use and alcohol-use disorders. Lancet 2009; 373: 2223-2233.

2 Spanagel R, Noori HR, Heilig M. Stress and alcohol interactions: animal studies and clinical significance. Trends Neurosci 2014; 37: 219-227.

3 Koob GF. Theoretical frameworks and mechanistic aspects of alcohol addiction: alcohol addiction as a reward deficit disorder. Curr Top Behav Neurosci 2013; 13: 3-30.

4 Contet C. Gene expression under the influence: transcriptional profiling of ethanol in the brain. Curr Psychopharmacol 2012; 1: 301-314.

5 Porrino $\mathrm{L}$, Whitlow CT, Samson HH. Effects of the self-administration of ethanol and ethanol/sucrose on rates of local cerebral glucose utilization in rats. Brain Res 1998; 791: 18-26.

6 Vilpoux C, Warnault V, Pierrefiche O, Daoust M, Naassila M. Ethanol-sensitive brain regions in rat and mouse: a cartographic review, using immediate early gene expression. Alcohol Clin Exp Res 2009; 33: 945-969.

7 Spanagel R. Alcoholism: a systems approach from molecular physiology to addictive behavior. Physiol Rev 2009; 89: 649-705.

8 Robison AJ, Nestler EJ. Transcriptional and epigenetic mechanisms of addiction. Nat Rev Neurosci 2011; 12: 623-637.

9 Sari Y, Johnson VR, Weedman JM. Role of the serotonergic system in alcohol dependence: from animal models to clinics. Prog Mol Biol Transl Sci 2011; 98: 401-443.

10 Marcinkiewcz CA. Serotonergic systems in the pathophysiology of ethanol dependence: relevance to clinical alcoholism. ACS Chem Neurosci 2015; 6: 1026-1039.

11 Koob GF, Volkow ND. Neurocircuitry of addiction. Neuropsychopharmacology 2010; 35: 217-238.

12 Brand I, Fliegel S, Spanagel R, Noori HR. Global ethanol-induced enhancements of monoaminergic neurotransmission: a meta-analysis study. Alcohol Clin Exp Res 2013; 37: 2048-2057.

13 Healey JC, Winder DG, Kash TL. Chronic ethanol exposure leads to divergent control of dopaminergic synapses in distinct target regions. Alcohol 2008; 42: 179-190.

14 Budygin EA, John CE, Mateo Y, Daunais JB, Friedman DP, Grant KA et al. Chronic ethanol exposure alters presynaptic dopamine function in the striatum of monkeys: a preliminary study. Synapse 2003; 50: 266-268.

15 Brodie MS. Increased ethanol excitation of dopaminergic neurons of the ventral tegmental area after chronic ethanol treatment. Alcohol Clin Exp Res 2002; 26: 1024-1030.

16 Ollat $\mathrm{H}$, Parvez $\mathrm{H}$, Parvez $\mathrm{S}$. Alcohol and central neurotransmission. Neurochem Int 1988; 13: 275-300.

17 Rimondini R, Arlinde C, Sommer W, Heilig M. Long-lasting increase in voluntary ethanol consumption and transcriptional regulation in the rat brain after intermittent exposure to alcohol. FASEB J 2002; 16: 27-35.

18 Jonsson S, Ericson M, Soderpalm B. Modest long-term ethanol consumption affects expression of neurotransmitter receptor genes in the rat nucleus accumbens. Alcohol Clin Exp Res 2014; 38: 722-729.

19 Volkow ND, Wang GJ, Fowler JS, Tomasi D. Addiction circuitry in the human brain. Annu Rev Pharmacol Toxicol 2012; 52: 321-336.

20 Ducci F, Goldman D. Genetic approaches to addiction: genes and alcohol. Addiction 2008; 103: 1414-1428.

21 De Bellis MD. Developmental traumatology: a contributory mechanism for alcohol and substance use disorders. Psychoneuroendocrinology 2002; 27: 155-170.

22 Becker HC, Lopez MF, Doremus-Fitzwater TL. Effects of stress on alcohol drinking: a review of animal studies. Psychopharmacology 2011; 218: 131-156.

23 Meaney MJ. Maternal care, gene expression, and the transmission of individual differences in stress reactivity across generations. Annu Rev Neurosci 2001; 24: 1161-1192.

24 Molet J, Maras PM, Avishai-Eliner S, Baram TZ. Naturalistic rodent models of chronic early-life stress. Dev Psychobiol 2014; 56: 1675-1688. 
25 Spivey JM, Padilla E, Shumake JD, Gonzalez-Lima F. Effects of maternal separation early handling, and gonadal sex on regional metabolic capacity of the preweanling rat brain. Brain Res 2011; 1367: 198-206.

26 Suri D, Teixeira CM, Cagliostro MK, Mahadevia D, Ansorge MS. Monoaminesensitive developmental periods impacting adult emotional and cognitive behaviors. Neuropsychopharmacology 2015; 40: 88-112.

27 Maccari S, Krugers HJ, Morley-Fletcher S, Szyf M, Brunton PJ. The consequences of early-life adversity: neurobiological, behavioural and epigenetic adaptations. J Neuroendocrinol 2014; 26: 707-723.

28 Enoch MA. The role of early life stress as a predictor for alcohol and drug dependence. Psychopharmacology 2011; 214: 17-31.

29 Barr CS. Non-human primate models of alcohol-related phenotypes: the influence of genetic and environmental factors. Curr Top Behav Neurosci 2013; 13: 223-249.

30 Enoch MA. Genetic and environmental influences on the development of alcoholism: resilience vs. risk. Ann NY Acad Sci 2006; 1094: 193-201.

31 Shih JC, Chen K, Ridd MJ. Monoamine oxidase: from genes to behavior. Annu Rev Neurosci 1999; 22: 197-217.

32 Berry MD, Juorio AV, Paterson IA. The functional role of monoamine oxidases A and $B$ in the mammalian central nervous system. Prog Neurobiol 1994; 42: 375-391.

33 Brunner HG, Nelen M, Breakefield XO, Ropers HH, van Oost BA. Abnormal behavior associated with a point mutation in the structural gene for monoamine oxidase A. Science 1993; 262: 578-580.

34 Denney RM, Koch H, Craig IW. Association between monoamine oxidase A activity in human male skin fibroblasts and genotype of the MAOA promoter-associated variable number tandem repeat. Human Genet 1999; 105: 542-551.

35 Sabol SZ, Hu S, Hamer D. A functional polymorphism in the monoamine oxidase A gene promoter. Human Genet 1998; 103: 273-279.

36 Fowler JS, Alia-Klein N, Kriplani A, Logan J, Williams B, Zhu W et al. Evidence that brain MAO A activity does not correspond to MAO A genotype in healthy male subjects. Biol Psychiatry 2007; 62: 355-358.

37 Shumay E, Logan J, Volkow ND, Fowler JS. Evidence that the methylation state of the monoamine oxidase A (MAOA) gene predicts brain activity of MAO A enzyme in healthy men. Epigenetics 2012; 7: 1151-1160.

38 Cervera-Juanes R, Wilhem L, Park B, Lee R, Locke J, Helms C et al. MAOA expression predicts vulnerability for alcohol use. Mol Psychiatry advance online publication, 7 July 2015; doi:10.1038/mp.2015.93

39 Oreland L, Nilsson K, Damberg M, Hallman J. Monoamine oxidases: activities, genotypes and the shaping of behaviour. J Neural Transm 2007; 114: 817-822.

40 Nilsson KW, Sjoberg RL, Damberg M, Leppert J, Ohrvik J, Alm PO et al. Role of monoamine oxidase A genotype and psychosocial factors in male adolescent criminal activity. Biol Psychiatry 2006; 59: 121-127.

41 Caspi A, McClay J, Moffitt TE, Mill J, Martin J, Craig IW et al. Role of genotype in the cycle of violence in maltreated children. Science 2002; 297: 851-854.

42 Guindalini C, Scivoletto S, Ferreira RG, Nishimura A, Zilberman ML, Peluso MM et al. Association of MAO A polymorphism and alcoholism in Brazilian females. Psychiatr Genet 2005; 15: 141-144.

43 Contini V, Marques FZ, Garcia CE, Hutz MH, Bau CH. MAOA-uVNTR polymorphism in a Brazilian sample: further support for the association with impulsive behaviors and alcohol dependence. Am J Med Genet B Neuropsychiatr Genet 2006; 141B: 305-308.

44 Nilsson KW, Sjoberg RL, Wargelius HL, Leppert J, Lindstrom L, Oreland L. The monoamine oxidase A (MAO-A) gene, family function and maltreatment as predictors of destructive behaviour during male adolescent alcohol consumption. Addiction 2007; 102: 389-398.

45 Ducci F, Enoch MA, Hodgkinson C, Xu K, Catena M, Robin RW et al. Interaction between a functional MAOA locus and childhood sexual abuse predicts alcoholism and antisocial personality disorder in adult women. Mol Psychiatry 2008; 13: 334-347.

46 Nilsson KW, Wargelius HL, Sjoberg RL, Leppert J, Oreland L. The MAO-A gene, platelet MAO-B activity and psychosocial environment in adolescent female alcohol-related problem behaviour. Drug Alcohol Depend 2008; 93: 51-62.

47 Nilsson KW, Comasco E, Aslund C, Nordquist N, Leppert J, Oreland L. MAOA genotype, family relations and sexual abuse in relation to adolescent alcohol consumption. Addict Biol 2011; 16: 347-355.

48 Ducci F, Newman TK, Funt S, Brown GL, Virkkunen M, Goldman D. A functional polymorphism in the MAOA gene promoter (MAOA-LPR) predicts central dopamine function and body mass index. Mol Psychiatry 2006; 11: 858-866.

49 Samochowiec J, Lesch KP, Rottmann M, Smolka M, Syagailo YV, Okladnova O et al. Association of a regulatory polymorphism in the promoter region of the monoamine oxidase A gene with antisocial alcoholism. Psychiatr Res 1999; 86: 67-72.

50 Newman TK, Syagailo YV, Barr CS, Wendland JR, Champoux M, Graessle M et al. Monoamine oxidase A gene promoter variation and rearing experience influences aggressive behavior in rhesus monkeys. Biol Psychiatry 2005; 57: 167-172.

51 Barr CS, Schwandt ML, Newman TK, Higley JD. The use of adolescent nonhuman primates to model human alcohol intake: neurobiological, genetic, and psychological variables. Ann NY Acad Sci 2004; 1021: 221-233.
52 Haberstick BC, Lessem JM, Hewitt JK, Smolen A, Hopfer CJ, Halpern CT et al. MAOA genotype, childhood maltreatment, and their interaction in the etiology of adult antisocial behaviors. Biol Psychiatry 2014; 75: 25-30.

53 Goldman D, Rosser AA. MAOA-environment interactions: results may vary. Biol Psychiatry 2014; 75: 2-3.

54 Koller G, Bondy B, Preuss UW, Bottlender M, Soyka M. No association between a polymorphism in the promoter region of the MAOA gene with antisocial personality traits in alcoholics. Alcohol Alcohol 2003; 38: 31-34.

55 Pautassi RM, Camarini R, Quadros IM, Miczek KA, Israel Y. Genetic and environmental influences on ethanol consumption: perspectives from preclinical research. Alcohol Clin Exp Res 2010; 34: 976-987.

56 Nylander I, Roman E. Is the rodent maternal separation model a valid and effective model for studies on the early-life impact on ethanol consumption? Psychopharmacology (Berl) 2013; 229: 555-569.

57 Gustafsson L, Nylander I. Time-dependent alterations in ethanol intake in male wistar rats exposed to short and prolonged daily maternal separation in a 4-bottle free-choice paradigm. Alcohol Clin Exp Res 2006; 30: 2008-2016.

58 Ruijter JM, Ramakers C, Hoogaars WM, Karlen Y, Bakker O, van den Hoff MJ et al. Amplification efficiency: linking baseline and bias in the analysis of quantitative PCR data. Nucleic Acids Res 2009; 37: e45.

59 Daoura L, Haaker J, Nylander I. Early environmental factors differentially affect voluntary ethanol consumption in adolescent and adult male rats. Alcohol Clin Exp Res 2011; 35: 506-515.

60 Vitalis T, Fouquet C, Alvarez C, Seif I, Price D, Gaspar P et al. Developmental expression of monoamine oxidases $\mathrm{A}$ and $\mathrm{B}$ in the central and peripheral nervous systems of the mouse. J Comp Neurol 2002; 442: 331-347.

61 Gaspar P, Cases O, Maroteaux L. The developmental role of serotonin: news from mouse molecular genetics. Nat Rev Neurosci 2003; 4: 1002-1012.

62 Cases O, Seif I, Grimsby J, Gaspar P, Chen K, Pournin S et al. Aggressive behavior and altered amounts of brain serotonin and norepinephrine in mice lacking MAOA. Science 1995; 268: 1763-1766.

63 Tzschentke TM, Schmidt WJ. Functional relationship among medial prefrontal cortex, nucleus accumbens, and ventral tegmental area in locomotion and reward. Crit Rev Neurobiol 2000; 14: 131-142.

64 Grunewald M, Johnson S, Lu D, Wang Z, Lomberk G, Albert PR et al. Mechanistic role for a novel glucocorticoid-KLF11 (TIEG2) protein pathway in stress-induced monoamine oxidase A expression. J Biol Chem 2012; 287: 24195-24206.

65 Marquez C, Poirier GL, Cordero Ml, Larsen MH, Groner A, Marquis J et al. Peripuberty stress leads to abnormal aggression, altered amygdala and orbitofrontal reactivity and increased prefrontal MAOA gene expression. Trans/ Psychiatry 2013; 3: e216.

66 Oreland L, Wiberg A, Winblad B, Fowler CJ, Gottfries CG, Kiianmaa K. The activity of monoamine oxidase $-A$ and $-B$ in brains from chronic alcoholics. J Neural Transm 1983; 56: 73-83.

67 Sherif F, Wahlstrom G, Oreland L. Brain GABA-transaminase and monoamine oxidase after chronic ethanol treatment in rats. Alcohol Clin Exp Res 1993; 17: 1313-1318.

68 Wiberg A, Wahlstroom G, Oreland L. Brain monoamine oxidase activity after chronic ethanol treatment of rats. Psychopharmacology 1977; 52: 111-113.

69 Fils-Aime ML, Eckardt MJ, George DT, Brown GL, Mefford I, Linnoila M. Early-onset alcoholics have lower cerebrospinal fluid 5-hydroxyindoleacetic acid levels than late-onset alcoholics. Arch Gen Psychiatry 1996; 53: 211-216.

70 Sher L, Oquendo MA, Grunebaum MF, Burke AK, Huang YY, Mann JJ. CSF monoamine metabolites and lethality of suicide attempts in depressed patients with alcohol dependence. Eur Neuropsychopharmacol 2007; 17: 12-15.

71 Oreland S, Raudkivi K, Oreland L, Harro J, Arborelius L, Nylander I. Ethanol-induced effects on the dopamine and serotonin systems in adult Wistar rats are dependent on early-life experiences. Brain Res 2011; 1405: 57-68.

72 Gongwer MA, Murphy JM, McBride WJ, Lumeng L, Li TK. Regional brain contents of serotonin, dopamine and their metabolites in the selectively bred high- and low-alcohol drinking lines of rats. Alcohol 1989; 6: 317-320.

73 Murphy JM, McBride WJ, Lumeng L, Li TK. Regional brain levels of monoamines in alcohol-preferring and -nonpreferring lines of rats. Pharmacol Biochem Behav 1982; 16: 145-149.

74 Murphy JM, McBride WJ, Lumeng L, Li TK. Contents of monoamines in forebrain regions of alcohol-preferring (P) and -nonpreferring (NP) lines of rats. Pharmacol Biochem Behav 1987; 26: 389-392.

75 Banki CM. Factors influencing monoamine metabolites and tryptophan in patients with alcohol dependence. J Neural Transm 1981; 50: 89-101.

76 Roman E, Nylander I. The impact of emotional stress early in life on adult voluntary ethanol intake-results of maternal separation in rats. Stress 2005; 8: 157-174.

77 Momeni S, Roman E. Subgroup-dependent effects of voluntary alcohol intake on behavioral profiles in outbred Wistar rats. Behav Brain Res 2014; 275: 288-296.

78 Crabbe JC, Harris RA, Koob GF. Preclinical studies of alcohol binge drinking. Ann NY Acadof Sci 2011; 1216: 24-40. 
79 Palm S, Nylander I. Alcohol-induced changes in opioid peptide levels in adolescent rats are dependent on housing conditions. Alcohol Clin Exp Res 2014; 38: 2978-2987.

80 Simms JA, Steensland P, Medina B, Abernathy KE, Chandler LJ, Wise R et al. Intermittent access to $20 \%$ ethanol induces high ethanol consumption in LongEvans and Wistar rats. Alcohol Clin Exp Res 2008; 32: 1816-1823.

81 Rhodes JS, Best K, Belknap JK, Finn DA, Crabbe JC. Evaluation of a simple model of ethanol drinking to intoxication in C57BL/6J mice. Physiol Behav 2005; 84: 53-63.

82 Becker HC. Animal models of excessive alcohol consumption in rodents. Curr Top Behav Neurosci 2013; 13: 355-377.

83 Wise RA. Voluntary ethanol intake in rats following exposure to ethanol on various schedules. Psychopharmacologia 1973; 29: 203-210.

84 Carnicella S, Ron D, Barak S. Intermittent ethanol access schedule in rats as a preclinical model of alcohol abuse. Alcohol 2014; 48: 243-252.

85 Liu W, Crews FT. Adolescent intermittent ethanol exposure enhances ethanol activation of the nucleus accumbens while blunting the prefrontal cortex responses in adult rat. Neuroscience 2015; 293: 92-108.

86 Roman E, Ploj K, Nylander I. Maternal separation has no effect on voluntary ethanol intake in female Wistar rats. Alcohol 2004; 33: 31-39.

87 Toffoletto S, Lanzenberger R, Gingnell M, Sundstrom-Poromaa I, Comasco E. Emotional and cognitive functional imaging of estrogen and progesterone effects in the female human brain: a systematic review. Psychoneuroendocrinology 2014; 50: $28-52$.

88 Barth C, Villringer A, Sacher J. Sex hormones affect neurotransmitters and shape the adult female brain during hormonal transition periods. Front Neurosci 2015; 9: 37.

89 Gundlah C, Lu NZ, Bethea CL. Ovarian steroid regulation of monoamine oxidase-A and $-\mathrm{B}$ mRNAs in the macaque dorsal raphe and hypothalamic nuclei. Psychopharmacology 2002; 160: 271-282.

90 Nakagawa S. A farewell to Bonferroni: the problems of low statistical power and publication bias. Behav Ecol 2004; 15: 1044-1045.

91 Perneger TV. What's wrong with Bonferroni adjustments. Br Med J 1998; 316: 1236-1238.

(c) (i) This work is licensed under a Creative Commons Attribution 4.0 International License. The images or other third party material in this article are included in the article's Creative Commons license, unless indicated otherwise in the credit line; if the material is not included under the Creative Commons license, users will need to obtain permission from the license holder to reproduce the material. To view a copy of this license, visit http://creativecommons.org/licenses/ by/4.0/

Supplementary Information accompanies the paper on the Translational Psychiatry website (http://www.nature.com/tp) 